\title{
NEONATAL STROKE DUE TO VARICELLA ARTERIOPATHY
}

\author{
Sampath Kumar N.S. ${ }^{1}$, Umamaheswara Reddy V. ${ }^{2}$, Amit Agrawal ${ }^{3}$, Ganesh V. ${ }^{1}$, \\ Mithilesh A. ${ }^{2}$ \\ ${ }^{1}$ Department of Neurology, Narayana Medical College Hospital, Chinthareddypalem, \\ Nellore, Andhra Pradesh, India \\ ${ }^{2}$ Department of Radiology, Narayana Medical College Hospital, Chinthareddypalem, \\ Nellore, Andhra Pradesh, India \\ ${ }^{3}$ Department of Neurosurgery, Narayana Medical College Hospital, Chinthareddypalem, \\ Nellore, Andhra Pradesh, India
}

\begin{abstract}
Varicella zoster is one of the common infective etiological factors known to produce arteriopathy of cerebral vasculature. Varicella zoster infection spreads transaxonally first to the adventitia and later transmurally to intima. Primary infection or reactivation of varicella zoster, both are associated with involvement of cerebral vessels of large as well as of small calibre varicella zoster associated arteriopathy was reported up to 12 months after the infection. Recurrence of strokes are seen post varicella infection. Hence the identification of varicella zoster as an etiological agent of ischemic stroke in neonatal age group patients is necessary and requires follow-up. Other neurologic sequelae following varicella infection are aneurysm formation leading to subarachnoid and cerebral haemorrhage, carotid dissection and rarely peripheral arterial disease. Due to lack of adequate epidemiological, neuroimaging and laboratory data, guidelines for treatment of varicella ateriopathy are not clear.
\end{abstract}

Keywords: post varicella arteriopathy, varicella zoster, arterial stenoses, middle cerebral artery, post varicella sequelae, seizures

\section{INTRODUCTION}

Arterial ischemic stroke (AIS) in neonates is of varied etiologies. Varicella zoster is recognized as one of the common infective etiological factors known to produce arteriopathy of cerebral vasculature. (1-3) Varicella zoster, a DNA virus, belonging to herpes viridae is the etiological agent of varicella (chickenpox) and herpes zoster. Mode of infection is through the aerosols or contact with the vesicular fluid. After the primary infection of Varicella (chickenpox), the virus becomes latent and persists in the regional ganglia for indefinite period only to reacti- vate when the person's immunity falls and manifests as Herpes zoster. (2). Primary infection or the reactivation of varicella zoster both are associated with involvement of cerebral vessels of large as well as of small calibre. (2,4-6) Varicella zoster associated arteriopathy (VZA) was reported upto 12 months after the infection. (7) Recurrence of strokes are seen post VZA. Hence the identification of varicella zoster as an etiological agent of AIS in paediatric age group patients is necessary to institute necessary treatment and requires follow periodically (7) Here we describe a case of VZA in a 10 month old which occured 2 months following chicken pox. 


\section{CASE REPORT}

A 10 months old child came to the Neurology department with complaints of 3 to 4 episodes of abnormal movements in the form of deviation of mouth and repeated eye blinking with staring looks. History of fever with rash 2 months before the development of symptoms and was diagnosed as chicken pox. The baby was a full term baby born out of normal vaginal delivery with normal birth weight and there was no history of birth anoxia or seizures. He received immunization according to the national immunization schedule. His social, motor and language milestones were normal for his age. Patient developed jaundice on the third day after birth and he was admitted in Neonatal ICU for 1 day. Anthropometric measurements: baby's head circumference $48 \mathrm{~cm}$; chest circumference $47 \mathrm{~cm}$; weight $9 \mathrm{Kgs}$. On general examination patient was anaemic ( $\mathrm{Hb}: 7.5 \%$ ), afebrile, conscious and alert. Vitals: Pulse rate was $140 / \mathrm{min}$, regular and all peripheral pulses felt. Blood pressure: 90/60mm of $\mathrm{Hg}$; Respiratory rate $24 / \mathrm{min}$. Neurological examination was normal except for the left facial palsy of UMN type and weakness of left upper and lower limbs. Power in left upper limb 2/5 and lower limb $3 / 5$. No abnormal movements were observed during the period of examination. Examination of other systems was found to be normal. MRI brain, brain angiogram and neck angiogram were performed which showed acute infarct in the right MCA territory. MR angiogram revealed M2 segment focal stenosis. MR neck angiogram did not reveal any abnormality. Haemogram was suggestive of microcytic and hypochromic anaemia and there was no evidence of sickle cells or haemoparasites. Metabolic work up including blood sugars, serum electrolytes, renal function tests, liver function tests and serum homocysteine levels were within normal limits. Patient was investigated for thrombotic and connective tissue disease profile for the etiological work up and they were found to be negative. Lumbar puncture and CSF analysis was suggestive of pleocytosis with normal sugars. Anti VZV IgG antibody was positive. HSV PCR was negative. EEG was a normal sedated sleep EEG record - generalised 3-5 Hz with frequent sleep spindles, delta activity and theta activity during the record. 2D echo revealed no evidence of congenital heart diseases. Patient was managed with antiplatelets and acyclovir. There was remarkable improvement within two days.

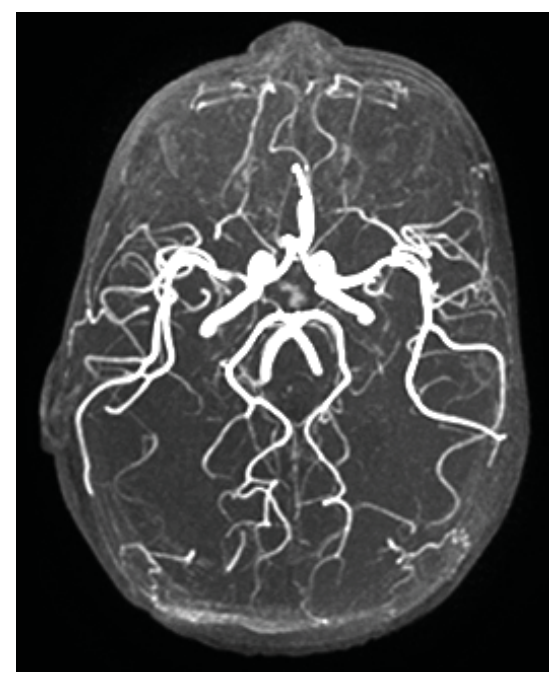

FIGURE 2. Non-contrast 3D TOF MR angiogram showing stenosis in the $\mathrm{M} 2$ segment of the right MCA

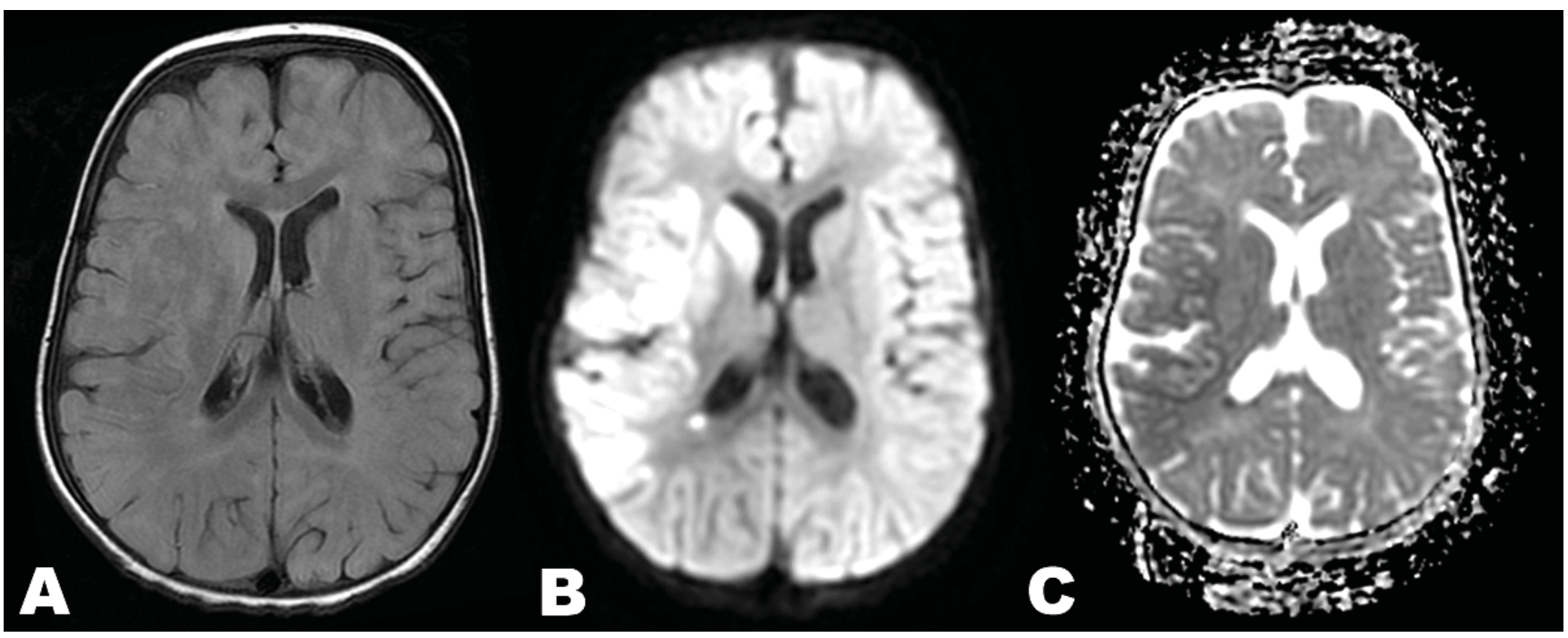

FIGURE 1. Axial FLAIR images (A) showing hyperintense area involving corona radiata, insular cortex and fronto-temporo-parietal gyri. Diffusion weighted images and corresponding ADC maps showing diffusion restriction in the areas of FLAIR hyperintensity suggestive of acute infarct. 


\section{DISCUSSION}

The most common arteriopathy associated with stroke in pediatric age group is transient cerebral arteriopathy (TCA). It is characterised by monophasic arterial disease manifested as unilateral focal or segmental stenosis, which involves the distal part of the internal carotid and the initial segments and branches of the anterior and/or middle cerebral arteries and is usually associated with complete or partial resolution. (1) According to Steiger et al, post varicella angiopathy is underdiagnosed and is one of the common causes of paediatric stroke. (8) The pathophysiology of TCA is clearly not known. Clinical manifestations of VZA in children are difficult to elicit. Irritability, seizures, abnormal movements and hemiparesis are common symptoms. $(2,4,9)$ Varicella zoster infection spreads transaxonally first to adventitia, later transmurally to intima. Characteristic histology of affected vessels in post varicella infection are: 1) breech in internal elastic lamina; 2) a hyperproliferating intima with cells expressing $\alpha$-smooth muscle actin and smooth muscle myosin heavy chain; 3) medial smooth muscle cell loss. Stroke in VZA may result in changes in arterial calibre and elasticity owing to loss of smooth muscles and abnormal accumulation of myofibroblasts. $(2,5,8)$ VZA can cause ischaemic infarction of the brain, spinal cord, as well as aneurysm formation leading to subarachnoid and cerebral haemorrhage, carotid dissection and rarely peripheral arterial disease. $(2-8,10)$ In the present case the median time interval from the varicella infection to arterial ischaemic stroke was 2 months. $(1,2,5)$ The presentation was with seizures followed by development of hemiparesis on left side. Due to lack of adequate epidemiological, neuroimaging and laboratory data, guidelines for treatment of varicella ateriopathy are not clear. Some expert neurologists advise anticoagulant and antiplatelet therapies like any other stroke, other group advises to treat the disease with antiviral and immunosuppressive medications. $(2,6,8)$ We managed the patient with antiepileptics and antiplatelets. The patient made a remarkable recovery in the next 2 weeks. The child was kept under supervision with regular follow-up visits to identify the resolution of arteriopathy and recurrence of symptoms.

\section{CONCLUSION}

Post varicella arteriopathy can cause ischaemic stroke in neonatal age group. The accurate diagnosis is important for the effective treatment and to prevent the recurrence of strokes. Guidelines for treatment of varicella arteriopathy are not clear. The VZA cases are managed with acyclovir and steroids or antiplatelets. We managed the patient successfully with antiepileptics and antiplatelets, 2 month follow-up showed no recurrence of symptoms.

\section{REFERENCES}

1. Dunkhase-Heinl U., Stausbøl-Grøn B., Christensen J., Ostergaard J.R. - Post-Varicella Angiopathy: A Series of 4 Patients With Focus on Virologic and Neuroimaging Findings. Pediatric Neurology: 50:581-585.

2. Nagel M.A., Gilden D. - Update on varicella zoster virus vasculopathy. Current infectious disease reports 2014; 16:407.

3. Selvakumar C.J., Justin C., Gnaneswaran T.R., Chandrasekaran M. - Post varicella angiopathy. The Journal of the Association of Physicians of India 2010; 58:572-574.

4. Girija A., Rafeeque M., Abdurehman K. - Neurological complications of chickenpox. Annals of Indian Academy of Neurology 2007; 10:240.

5. Lanthier S., Armstrong D., Domi T. - Post-varicella arteriopathy of childhood Natural history of vascular stenosis. Neurology 2005; 64:660-663.

6. Nagel M.A., Mahalingam R., Cohrs R.J., Gilden D. - Virus vasculopathy and stroke: an under-recognized cause and treatment target. Infectious disorders drug targets 2010; 10:105-111.

7. Hayes B., Baker L., Alhajeri A., Ryan S., Lynch B. - Ischaemic stroke in children secondary to post varicella angiopathy. Irish medical journal 2007; 100:332-333.

8. Steiger H.-J., Hänggi D., Assmann B., Turowski B. - Cerebral angiopathies as a cause of ischemic stroke in children: differential diagnosis and treatment options. Deutsches Ärzteblatt International 2010; 107:851.

9. Nagel M., Cohrs R., Mahalingam R., et al. - The varicella zoster virus vasculopathies Clinical, CSF, imaging, and virologic features. Neurology 2008; 70:853-860.

10. Yaramış A., Hergüner S., Kara B., Tatı B., Tüzün Ü., Özmen M. Cerebral vasculitis and obsessive-compulsive disorder following varicella infection in childhood. The Turkish journal of pediatrics 2009; 51:072-075. 